\title{
Application of Attribute Based Access Control Model for Industrial Control Systems
}

\author{
Erkan Yalcinkaya \\ Department of Production Engineering, Royal Institute of Technology, Stockholm, Sweden \\ E-mail: erkany@kth.se \\ Antonio Maffei and Mauro Onori \\ Department of Production Engineering, Royal Institute of Technology, Stockholm, Sweden \\ E-mail: \{maffei, onori\}@kth.se
}

\begin{abstract}
The number of reported security vulnerabilities and incidents related to the industrial control systems (ICS) has increased recent years. As argued by several researchers, authorization issues and poor access control are key incident vectors. The majority of ICS are not designed security in mind and they usually lack strong and granular access control mechanisms. The attribute based access control (ABAC) model offers high authorization granularity, central administration of access policies with centrally consolidated and monitored logging properties. This research proposes to harness the ABAC model to address the present and future ICS access control challenges. The proposed solution is also implemented and rigorously tested to demonstrate the feasibility and viability of ABAC model for ICS.
\end{abstract}

Index Terms - Attribute based access control (ABAC), industrial control systems (ICS), fine grained authorization, central policy enforcement.

\section{INTRODUCTION}

Industrial control systems (ICS) are designed to automate and control a wide range of industrial processes such as manufacturing, water supply, power generation, transportation and so forth.

ICS can be in different forms for instance programmable logic controllers (PLC), supervisory control and data acquisition systems (SCADA) and distributed control systems (DCS). Many of these controlling units were initially designed for fairly specific tasks which were assumed to be performed in isolated environments like factory production lines. However, since the industry 4.0 revolution, ICS have become more versatile to perform diverse tasks. Moreover, due to the rapid increase in computational power, ICS has evolved into programmable interconnected computers rather than individual units.

The era of interconnection has brought ICS to the forefront of cyber security. Therefore, the reported number of incidents related to ICS has increased in the last five years. Fig. 1 shows the number of "ICS related vulnerability reports - tickets" reported between the year 2010 and 2013 [1][2].
According to the research published by the U.S. Department of Homeland Security [3], poor access control and misconfigured authorization schemas were the second and the third highest percentage of ICS vulnerabilities identified between 2009 and 2010.

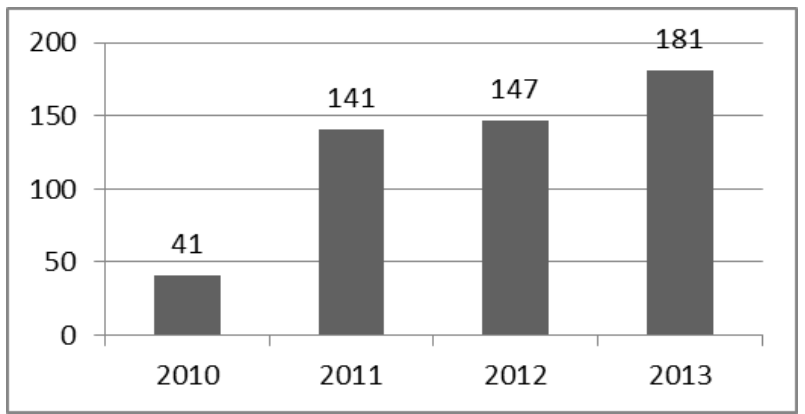

Fig.1. - ICS Related Vulnerability Reports - Tickets between the Year 2010 and 2013

Likewise, the report published by ICS-CERT [4] highlights the unauthorized access issues with interconnected ICS/SCADA devices. The same report also identifies "Abuse of access authority" and "Weak authentication" as the key incident vectors of the year 2014.

As GE Measurement \& Control Solutions [5] and National American Reliability Council [6] state, authorization, authentication and access control vulnerabilities are the most common vulnerabilities identified in the ICS domain.

As stated above, many ICS related security risks are due to the insufficient, misconfigured or vulnerable access control mechanisms. Therefore, in order to address those weaknesses, this research proposes to harness the attribute based access control (ABAC) model for ICS.

\section{ACCESS CONTROL MECHANISM}

In the context of information security, access control mechanisms ensure that subjects are allowed to perform only authorized operations on objects in question. In other words, access control mechanisms enforce confidentiality [7, p. 2], integrity [7, p. 3] and indirectly availability [7, p. 4] of a given protected object. 
The access control paradigm describes the objects as resources (for instance hardware, sensor, robot, database table etc.) and subjects as entities initiating the access request. The same paradigm also describes [9] number of security services namely authentication (confirmation of subject's identity [7, p. 171]), authorization (granted set of permissions to perform certain type of actions) and auditing (accountability by monitoring subject's activities [7, p. 423]) to achieve the desired access protection.

\section{TRAditional ACCESS CONTROL MODELS}

The most prominent traditional access control models are Mandatory Access Control (MAC) Model, Discretionary Access Control (DAC) Model and RoleBased Access Control (RBAC) Model. The following three subsections briefly elaborate these models.

\section{A. MAC Model}

The MAC (a.k.a. Rule based) model compares the subject's clearance with the object's classification level. The access decision is given by the operating system which is considered as a black box and cannot be influenced by the end users [6, p. 53]. Military IT systems often rely on MAC.

\section{B. DAC Model}

In contrast to the MAC, the DAC (a.k.a. Identity-based) model allows the object owners to set/control the access rules (via access control lists) [6, p. 53].

\section{RBAC Model}

The RBAC (a.k.a. nondiscretionary access control) model defines access groups which are set of privileges to perform a particular type of operation(s) [9]. The access groups are assigned to subjects and often determined by classification of job functions or responsibilities within the enterprise.

\section{Attribute Based Access Control Model}

The traditional access control models described in the previous sections focus only on subject's identity. Thus, the possible combinations of access conditions are quite limited.

The DAC model offers only basic level of security which can potentially be circumvented [10]. For instance, assume a subject who is granted read only access to a particular file. Even though the subject is prohibited any other operation then reading, he/she can copy the file content to another file which he/she might have full access to perform any operation [11].

The MAC model is pretty rigid and difficult to administrate [10]. Therefore it is impractical to utilize in contemporary transaction intense distributed IT systems.

The RBAC model well suits for small size systems, however, due to the cross referencing issue, the required number of roles for large enterprises may potentially exceed manageable levels [11].

These shortcomings of the traditional access control models are addressed by the attribute based access control (ABAC) model.

The ABAC model in a nutshell is based on five fundamental cornerstones (Fig. 2). These are subjectobject attributes, environment conditions, centrally administrated access policy and desired operation.

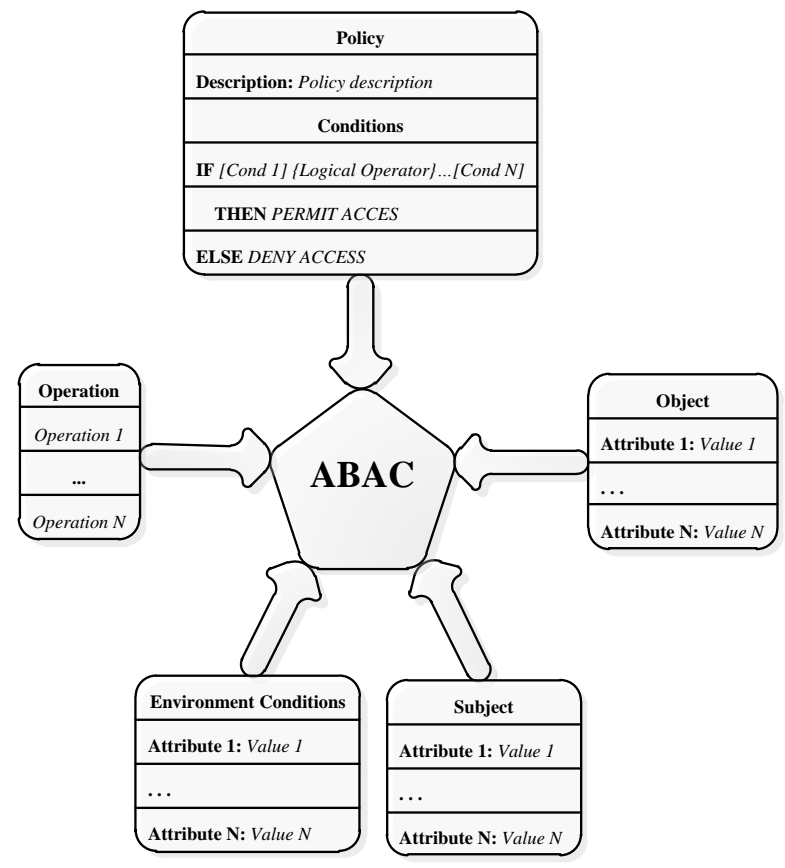

Fig.2. Five Cornerstones of ABAC: Policy, Subject, Object, Operation Type and Environment Conditions 
As visualized in Fig. 2, the access request is permitted or denied if all the policy conditions are met. The access policy validates the combinations of subject, object, and environment attribute values as well as the operation type. Therefore, the number of possible variations of distinct access conditions (i.e. access granularity) is significantly larger than the traditional access control models.

The versatility of ABAC model boils into its simplicity and flexibility to support fine-grained authorizations enforced by centrally administered access control policies. Therefore, the access control is logically decoupled from the business logic. Thus, both introducing a new policy and updating an existing one can easily be performed from the central policy repository without affecting the integrated systems.

The ABAC model is also capable of interpreting different threat levels as environment or subject attributes [12]. If the risk score is above a certain threshold, the access request can automatically be denied. For example, assume an access policy which identifies the risk threshold score as 5. If a subject is authenticated with a single factor from the enterprise network, the risk assessment engine evaluates the risk score as 3 , and the access request is granted. However, if the subject is authenticated with a single factor from a remote location, the risk score is evaluated as 7 . Thus, the access request is denied. If the same subject is identified with a secondary means of authentication (multifactor authentication), then the risk score is lowered to 4 and the access request is granted.

In addition to all these advantages, the ABAC model is backwards compatible. In other words, it is also flexible and versatile enough to support proprietary systems designed to work with traditional access control models such as MAC, DAC and RBAC [9].

\section{NIST REFERENCE ABAC ARCHITECTURE}

There are number of recommended reference architectures addressing the ABAC model in an enterprise environment. However, this research focuses on the reference ABAC architectural framework described in the NIST Special Publication 800-162 [10]. Table 1 highlights the main components of the reference architecture.

Table 1. Architectural Components of NIST Reference ABAC Framework

\begin{tabular}{|c|c|}
\hline Component & Description \\
\hline Subject & An entity requesting access to a protected object entity \\
\hline Protected Object & A protected resource entity being requested for access \\
\hline Policy & A rule expressing the relationship between a subject and a protected object \\
\hline Attribute & Set of properties bound to a particular subject or a protected object \\
\hline Operation & A type of action that a subject attempts to perform on a protected object \\
\hline Environment Conditions & $\begin{array}{l}\text { A set of properties which are not bound to a subject or a protected object but } \\
\text { related to the context such as current date, time, place or risk level }\end{array}$ \\
\hline Policy Repository & An enterprise-wide central database of authorization policies \\
\hline Attribute Repository & An enterprise-wide central database of protected object and subject attributes \\
\hline Policy Decision Point (PDP) & $\begin{array}{l}\text { A central access decision engine which permits or denies the access requests by } \\
\text { evaluating the authorization policy along with the attributes of a subject, a } \\
\text { protected object and environment conditions }\end{array}$ \\
\hline Policy Enforcement Point (PEP) & $\begin{array}{l}\text { An interceptor mediating all access requests towards a specific protected object. } \\
\text { PEP queries PDP to validate if the subject in question is permitted or denied the } \\
\text { access request }\end{array}$ \\
\hline Policy Information Point (PIP) & $\begin{array}{l}\text { A data service interface towards PDP to fetch environment conditions, subject and } \\
\text { protected object attributes }\end{array}$ \\
\hline Policy Administration Point (PAP) & An external tool to manage authorization policies \\
\hline
\end{tabular}

As shown in Fig. 3, a subject requests permission from PEP to perform an operation on a protected object. Upon this, PEP queries PDP for the access decision. PDP then finds the corresponding access policy which may require relevant subject, object and/or environment attribute values (via PIP) to evaluate the final access decision. The outcome of the evaluation is then passed to PEP which ultimately "Denies" or "Permits" the requested operation. Last but not least, audit logs chronologically record all activities among different components of the ABAC system.

\section{ABAC MODEL FOR ICS}

The majority of the industrial control systems (ICS) are not designed security in mind and they usually depend on perimeter-based security tools like firewalls to split the secure internal factory networks from unsecure internet ecosystems. However, as [13] elaborates; this is a false sense of security. First of all, firewalls designed to block or permit only specific protocols (port numbers) therefore they can merely offer a limited level of security. Moreover, cross referencing firewall rules may easily become over complicated. According to [13], 80\% of the enterprise firewall installations are vulnerable due to 
misconfigured complex rules. The same research also indicates that the firewalls do not provide protection against insider threats originated by trusted partners, former or existing employees.

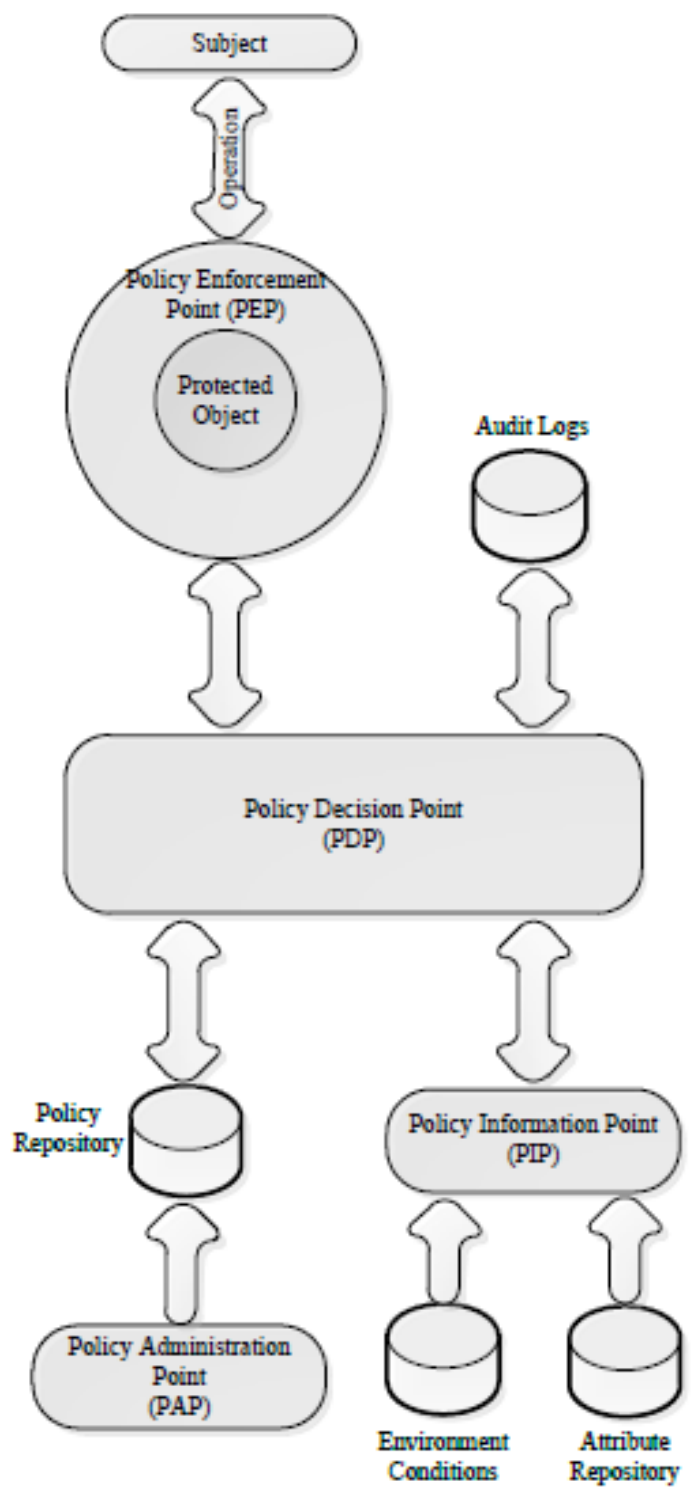

Fig.3. ABAC Reference Architecture

One way of addressing the insider threats is employing enterprise wide strict access control mechanisms. Thus, some researchers proposed ICS to harness RBAC model [14][15]. Although it is a step forward, due to flexibility and scalability issues as well as difficulties to implement fine-grained authorization rules and complexity in role administration (especially considering the complex system to system, human to system, and human to human interactions) for cross-referencing roles, the RBAC model is not the optimal access control solution for ICS.

The audit trail, records of all activities of a particular object during a given time interval, is another vital ICS security feature highlighted by NIST [16]. A comprehensive audit trail ensures accountability and is considered as one of the most important assets for performing forensics investigations. Thus, enterprise- wide audit records have to be centrally administered and secured. However, log consolidation is not straight forward for ICS, as they are scattered around the factory floor or even geographically remote locations.

Compliance and adherence to international standards, laws and company policies are imperative. When it comes to the ICS domain, ISA99 [17] and NIST Publication 800-82 [16] are the two foremost standards both of which have a dedicated chapter for controlling unauthorized access. However, due to the versatility and ubiquity, it has always been extremely challenging to prove and enforce the compliance requirements for the ICS-based ecosystems.

The industry 4.0 revolution has empowered cyber physical systems to highly interact with each other to increase the production efficiency. The improved ICS computational power will eventually allow the cyber physical systems to become more autonomous. As Evolvable production systems (EPS) concept describes [18], the future production systems will eventually be equipped with extraordinary skills like self-organization, self-diagnose and self-healing capabilities.

One of the biggest design challenges of EPS is controlling and restricting the interaction between autonomous systems. Therefore, EPS requires a flexible and highly scalable access control model to reach the ultimate goal of autonomy.

As mentioned in the previous sections, the $\mathrm{ABAC}$ model offers fine-grained, highly scalable, interoperable and flexible authorization model that is suitable for different layers of any given enterprise infrastructure. Moreover, regulatory compliance and auditing are streamlined by centrally enforced, managed and monitored policies. Likewise, the central management consolidates the audit $\operatorname{logs}$ and reduces the complexity of $\log$ management. Therefore, forensics investigations are substantially simplified. Furthermore, the ABAC model is backward compatible with the traditional access control models. In other words, proprietary ICS depending on DAC, MAC or RBAC model can be transparently converted to employ the ABAC model.

In conclusion, considering the features of the $\mathrm{ABAC}$ model along with the existing and the future ICS access control challenges, this research propose to utilize the ABAC model for ICS.

\section{ABAC MODEL FOR ICS USE CASE}

This section describes a use case to exemplify the proposed concept. The use case embodies authorization of an operator (human actor) to perform certain operations with the help of a PLC controlled robotic arm. Fig. 4 depicts the high-level relationship between the operator and the PLC-controlled robotic arm in the context of ABAC model.

For further clarification, it is worth materializing the use case with a real life scenario. Table 2 lists the attributes of sample subject-object couple along with environment conditions. Likewise, Table 3 defines a sample access policy. 


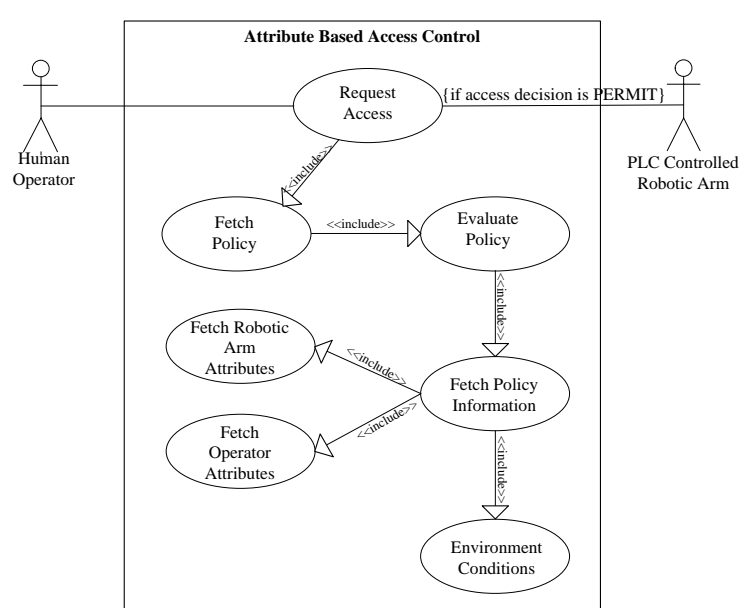

Fig.4. Use Case - A Human Operator and a PLC-Controlled Robotic Arm

Table 2. A Subject, Object and Environment Attribute Examples

\begin{tabular}{ll}
\hline \multicolumn{2}{c}{ Subject } \\
\hline Name & Anders \\
\hline Surname & Andersson \\
\hline $\begin{array}{l}\text { Date of } \\
\text { birth }\end{array}$ & 10.01 .1975 \\
\hline User ID & WR1000000 \\
\hline Department & $\mathrm{R} \& \mathrm{D}$ \\
\hline Roles & CNCOperator \\
\hline Emp. type & Permanent \\
\hline Clearance & Confidential \\
\hline
\end{tabular}

\begin{tabular}{|c|c|}
\hline \multicolumn{2}{|c|}{ Environment Conditions } \\
\hline Current date & 10.10 .2015 \\
\hline Current time & $10: 15$ \\
\hline Payload & $2 \mathrm{~kg}$ \\
\hline$I P$ & 10.0 .0 .15 \\
\hline $\begin{array}{l}\text { Comm. } \\
\text { protocol }\end{array}$ & TCP/IP \\
\hline Client type & $\begin{array}{l}\text { Corporate } \\
\text { PC }\end{array}$ \\
\hline $\begin{array}{l}\text { Encryption } \\
\text { Type }\end{array}$ & AES \\
\hline Threat level & Low \\
\hline
\end{tabular}

\begin{tabular}{ll}
\hline & Object \\
\hline Manufacturer & KUKA Robotics \\
\hline Model & KR 16-2 \\
\hline Asset ID & AT1000000 \\
\hline Department & Assembly Line \\
\hline Comp. type & Low payload robotic arm \\
\hline Classification & Confidential \\
\hline Function & Handling and Loading \\
\hline Max Reach & $1610 \mathrm{~mm}$ \\
\hline Number of Axes & 6 \\
\hline Max payload & $16 \mathrm{~kg}$ \\
\hline
\end{tabular}

Table 3. A Policy Example

\begin{tabular}{ll}
\hline \multicolumn{1}{c}{ Policy } \\
\hline Description & Evaluate robotic arm access request \\
\hline & IF [Object Department IS Assembly Line] AND \\
& {$[$ Subject clearance level IS HIGHER THAN OR } \\
& EQUAL TO Object classification level] AND \\
& {$[$ [Encryption type IS AES] AND } \\
Conditions & {$[$ IP IS Internal IP] AND } \\
& {$[$ Current time IS IN Working hours] AND } \\
& {$[$ Operation IS Pick] AND } \\
& {$[$ Payload IS LESS THAN 16kg] } \\
& THEN PERMIT ACCES \\
& ELSE DENY ACCESS \\
\hline
\end{tabular}

\begin{tabular}{|c|c|c|}
\hline Subject & \multirow[b]{2}{*}{ Operation } & Environment Conditions \\
\hline Name: Anders & & Current date: 20.11 .2016 \\
\hline \multicolumn{3}{|l|}{ Surname: Andersson } \\
\hline \multicolumn{3}{|l|}{ Date of birth: 10.01 .1975} \\
\hline \multicolumn{3}{|l|}{ User ID: WR1000000 } \\
\hline \multicolumn{3}{|r|}{ Comm. protocol: $T C P / I P$} \\
\hline \multicolumn{3}{|r|}{ Client type: Corporate $P C$} \\
\hline \multicolumn{3}{|l|}{ Employment type: Permanent } \\
\hline \multicolumn{3}{|l|}{ Clearance: Confidential } \\
\hline Object & & Policy \\
\hline Manufacturer: KUKA Robotics & Descript & te robotic arm access request \\
\hline \multicolumn{3}{|l|}{ Model: $K R$ 16-2 } \\
\hline \multicolumn{3}{|c|}{ IF [Object Department IS Assembly Line] AND } \\
\hline \multicolumn{3}{|c|}{ [Subject clearance level IS HIGHER THAN OR } \\
\hline \multicolumn{3}{|c|}{ EQUAL TO Object classification level] AND } \\
\hline \multicolumn{3}{|l|}{ Classification: Confidential } \\
\hline \multicolumn{3}{|l|}{ Function: Handling and Loading } \\
\hline \multicolumn{3}{|c|}{ [Current time IS IN Working hours] AND } \\
\hline \multicolumn{3}{|c|}{ [Operation IS Pick] AND } \\
\hline \multirow[t]{3}{*}{ Max payload: $16 \mathrm{~kg}$} & [Payle & S THAN $16 \mathrm{~kg}]$ \\
\hline & THEI & ACCES \\
\hline & ELSE $D$ & \\
\hline
\end{tabular}

Fig.5. ABAC Model for ICS Example with Policy Conditions, Subject, Object, Operation and Environment Attributes 
According to the use case; Anders, who is an operator, wishes to perform "Pick" operation with the help of PLCcontrolled robotic arm. As shown in Fig. 3, the policy enforcement point (PEP) logically protects the robotic arm from unauthorized access. After receiving the access request, PEP queries the policy decision point (PDP) with the requested operation, "Pick". Then PDP populates the five cornerstones of ABAC (Fig. 5). In other words, the access policy (Table 3) is fetched from the policy repository; the object, subject and environment attributes (Table 2) are fetched from the attribute repository via the policy information point (PIP). Subsequently, PDP evaluates the access policy and replies the access decision to the policy enforcement point (PEP) which then permits or denies the access request. Meanwhile, the central audit logging process in PDP updates the audit trail with relevant logging events.

In the scenario above, Anders belongs to the research and development department and his clearance level is higher than the classification of the robotic arm. Besides, he is located inside the enterprise network (internal IP) and the communication channel is secured with AES encryption. He requests to perform "Pick" operation for $2 \mathrm{~kg}$ payload during the working hours. Given all these facts, PDP decides to "Permit" the access request and PEP allows Anders to carry out the operation.

The sequence diagram shown in Fig. 6 illustrates the relationship among all components mentioned in the scenario above.

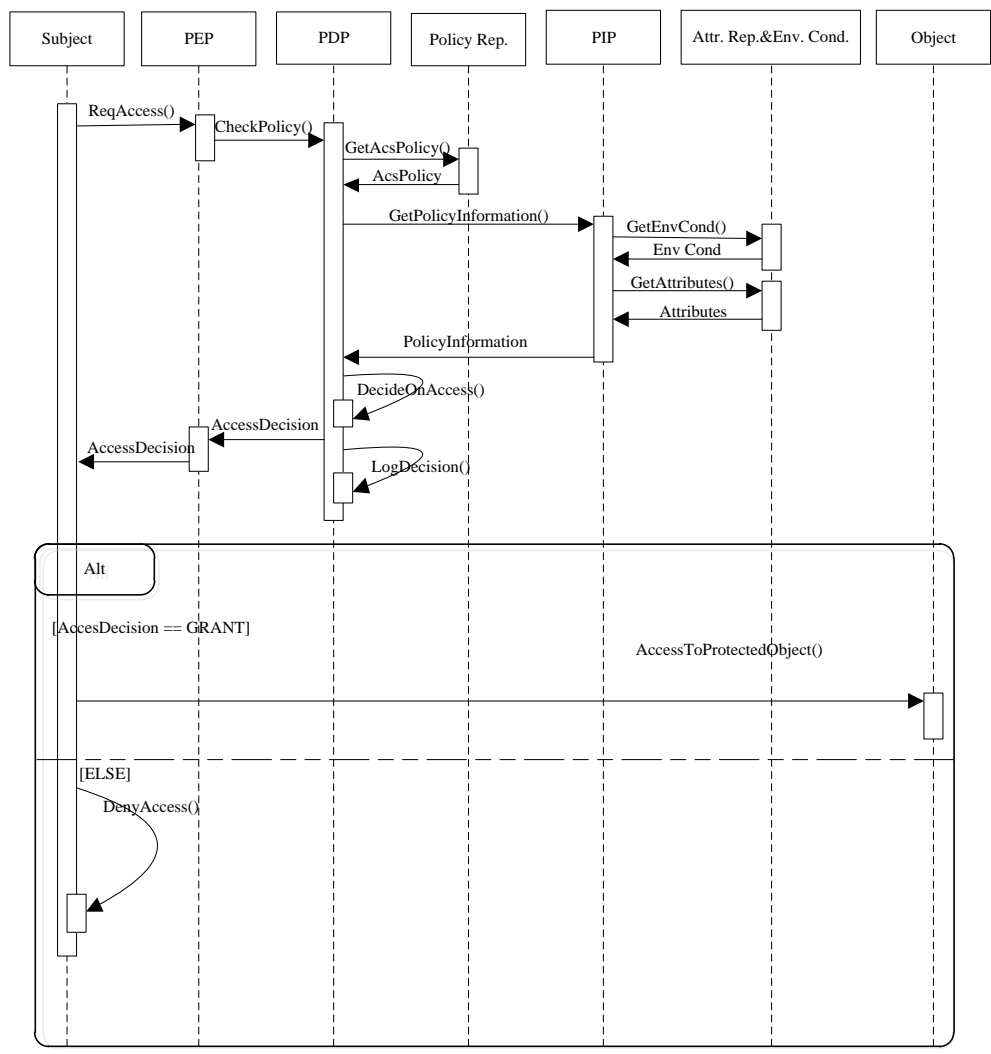

Fig.6. Sequence Diagram in the Context of Human Operator and PLC-Controlled Robotic Arm Authorization

\section{IMPLEMENTATION}

This section of the research elaborates the implementation details of the proposed scenario. The proof of concept setup is based on an open source framework, WSO2 Identity Server [19] and an open source relational database, MySQL [20]. Functional and load testing are performed with the help of another open source tool, Soap UI [21].

In the context of the $\mathrm{ABAC}$ reference architecture (Fig. 3), WSO2 Identity Server represents the policy decision point (PDP) and the policy repository, MySQL database functions (see Fig. 7 for Database Entity Relationship diagram) as the attribute repository for subject, object and environment conditions (see Appendix D for the definitions of the request and policy attributes). The custom developed (in Java) policy information point (PIP) fetches the relevant attribute values from the attribute repository. For the sake of simplicity, Soap UI assumed to represent the policy enforcement point (PEP). The prepared test cases simulate access requests (Soap format) towards PDP. Last but not least, the access rule (see Appendix B for the test policy) is implemented in eXtensible Access Control Markup Language (XACML). 


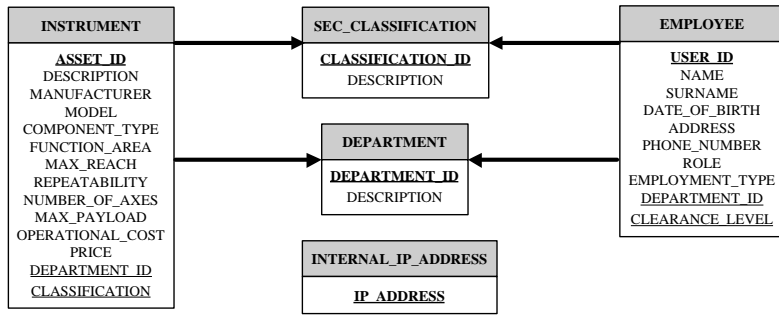

Fig. 7 - Database Entity Relationship Diagram

\section{TESTING}

The testing strategy comprises functional and load testing suites. As mentioned in the previous section, Soap UI is utilized for automating the testing procedure. The proof of concept setup is installed and configured on a Windows based office laptop with a quad-core desktop processor and 4GB of RAM.

The functional test suite has been formulated to cover positive and negative test cases. In other words, the correctness of deny and permit cases have been validated under various scenarios (see Appendix A for a sample Soap request resulting permit).

The load test suite has been formulated to measure the end to end average response time. The test suit blends a variety of successful and unsuccessful cases to simulate the real life scenario. Fig. 8 illustrates the average response time versus the total throughput (number of processed requests) for increasing number of concurrent requesting threads (from 1 to 100).

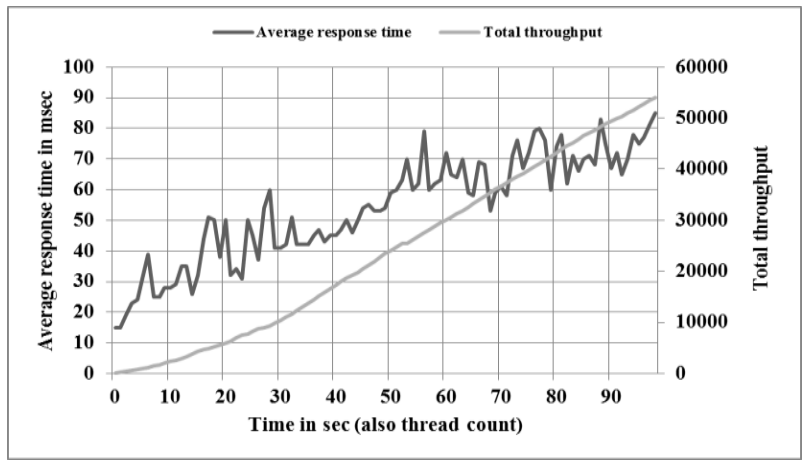

Fig.8. Average Response Time Compared Versus Total Throughput for Increasing Number of Concurrent Requesting Threads (From 1 to 100)

\section{Discussions}

The information security is fundamentally based on confidentiality, integrity and availability components. This research proposes to improve the confidentiality component for ICS by harnessing the ABAC model. However, the other two components are equally significant and improving one should not too much negatively affect the other two. In our case, introducing ABAC model for ICS apparently increases the computational burden which eventually affects the whole system's availability.

Although the latency requirement may vary for different types of ICS, it should be under a certain threshold to make sure that the criticality and responsiveness of the overall system are not compromised. According to Alcatel [23], the latency for real-time control and monitoring systems should be below 20 milliseconds whereas according to U.S. Department of Homeland Security [22], UTC and Avista [23], latency should not exceed 200 milliseconds.

In relation to our implementation; the end to end latency of the test setup reaches up to 85 milliseconds with roughly 500 request/sec throughput for 100 concurrent requesting threads (see Fig. 8). Given the low computational power of the test setup, the latency (availability) could still be considered at an acceptable level for many ICS.

Many of the ICS play a key role in automating and controlling core industrial processes, thus they are classified as business or even safety critical. Given their importance, system robustness and resilience are two fundamental design factors influencing the ICS based systems.

The proposed solution relies on a central decision point, PDP. Therefore PDP becomes a single point of failure and it has to be robust enough to tolerate the possible system faults. In other words, the access control manager has to be highly available and should employ redundancy.

The high availability and the low latency requirements can be met by distributing the system load among multiple instances of mirrored PDPs. Therefore, if one of the nodes is overloaded or down, the traffic could be diverted (failover) through the other instances.

\section{CONCLUSIONS}

The majority of ICS lack even basic security services like authentication and authorization as ICS are believed to be operating in isolated network segments inside a factory or production line and having perimeter-based security tools such as firewalls is good enough to keep ICS secure. However, an increasing number of ICS related security incidences contradicts the common belief.

The industrial production systems are foreseen to evolve into having self-organization, self-diagnose and self-healing capabilities in the near future. These new capabilities also create new challenges such as access control and management.

Being identified as one of the key ICS incident vectors, poor access control has been proposed to be addressed by some researchers with traditional access control models like RBAC. However, due to the complexity of role administration, scalability issues and difficulties to implement fine-grained authorization rules, RBAC model does not fully fit the ICS access control challenges.

$\mathrm{ABAC}$ is an emerging access control model offering fine-grained authorization with central access policy management and consolidated logging functionalities.

This research proposes to harness the ABAC model for ICS to address the present and future ICS access control challenges. The proposed solution is implemented and successfully tested for numerous scenarios. The test results proved the viability of the proposed solution with 
a satisfactory performance that might even be improved with distributed system architecture.

\section{APPENDIX A}

$<? \mathrm{xml}$ version="1.0"?>

<Request xmlns="urn:oasis:names:tc:xacml:3.0:core:schema:wd-17"

CombinedDecision="false" ReturnPolicyIdList="false">

$<$ Attributes Category="urn:oasis:names:tc:xacml:1.0:subject-

category:access-subject">

$<$ Attribute

AttributeId="urn:oasis:names:tc:xacml:1.0:subject:subject-id"

IncludeInResult="true">

$<$ AttributeValue

DataType="http://www.w3.org/2001/XMLSchema\#string">WR100000

$0</$ AttributeValue $>$

$</$ Attribute $>$

$</$ Attributes $>$

$<$ Attributes Category="urn:oasis:names:tc:xacml:3.0:attribute-

category:resource">

$<$ Attribute

AttributeId="urn:oasis:names:tc:xacml:1.0:resource:resource-id" IncludeInResult="true">

$<$ AttributeValue

DataType="http://www.w3.org/2001/XMLSchema\#string" $>$ AT100000 $<$

/AttributeValue>

$</$ Attribute>

$<$ Attribute AttributeId="http://kth.se/ics/departmentid"

IncludeInResult="true">

$<$ AttributeValue

DataType="http://www.w3.org/2001/XMLSchema\#string">100</Attrib

uteValue $>$

$</$ Attribute $>$

<Attribute AttributeId="http://kth.se/ics/payload"

IncludeInResult="true">

$<$ AttributeValue

DataType="http://www.w3.org/2001/XMLSchema\#integer" $>\mathbf{1 6}</$ Attrib uteValue>

$</$ Attribute $>$

$</$ Attributes $>$

$<$ Attributes Category="urn:oasis:names:tc:xacml:3.0:attribute-

category:action">

<Attribute AttributeId="urn:oasis:names:tc:xacml:1.0:action:action-

id" IncludeInResult="true">

$<$ AttributeValue

DataType="http://www.w3.org/2001/XMLSchema\#string" $>$ pick $</$ Attri

buteValue>

$</$ Attribute $>$

$</$ Attributes $>$

<Attributes Category="urn:oasis:names:tc:xacml:3.0:attribute-

category:environment">

<Attribute AttributeId="http://kth.se/ics/commencryptiontype" IncludeInResult="true">

$<$ AttributeValue

DataType="http://www.w3.org/2001/XMLSchema\#tstring" $>$ aes $</$ Attrib uteValue>

$</$ Attribute $>$

$<$ Attribute

AttributeId="urn:oasis:names:tc:xacml:1.0:environment:environment-

id" IncludeInResult="true">

$<$ AttributeValue

DataType="http://www.w3.org/2001/XMLSchema\#string"> 10.0.0.15</

AttributeValue $>$

$</$ Attribute $>$

$<$ Attribute

AttributeId="urn:oasis:names:tc:xacml:1.0:environment:current-time"

IncludeInResult="true">

$<$ AttributeValue

DataType="http://www.w3.org/2001/XMLSchema\#time">12:00:00</A

ttributeValue $>$

$</$ Attribute $>$

$</$ Attributes $>$

$</$ Request $>$

\section{APPENDIX B}

$<$ ?xml version="1.0"?>

$<$ Policy xmlns="urn:oasis:names:tc:xacml:3.0:core:schema:wd-17"

xmlns:xacml="urn:oasis:names:tc:xacml:3.0:core:schema:wd-17"

PolicyId="ICS-Policy"

RuleCombiningAlgId="urn:oasis:names:tc:xacml:1.0:rule-combiningalgorithm:first-applicable" Version="1.0">

$<$ Target/>

$<$ Rule RuleId="rule1" Effect="Permit" $>$

$<$ Target>

$<$ AnyOf $>$

$<$ AllOf $>$

$<$ Match MatchId="urn:oasis:names:tc:xacml:3.0:function:stringequal-ignore-case">

$<$ AttributeValue

DataType="http://www.w3.org/2001/XMLSchema\#string" >pick $</$ Attri buteValue>

$<$ AttributeDesignator MustBePresent="false"

Category="urn:oasis:names:tc:xacml:3.0:attribute-category:action"

Attributeld="urn:oasis:names:tc:xacml:1.0:action:action-id"

DataType="http://www.w3.org/2001/XMLSchema\#string"/> $</$ Match $>$

$<$ Match MatchId="urn:oasis:names:tc:xacml:3.0:function:string equal-ignore-case">

$<$ AttributeValue

DataType="http://www.w3.org/2001/XMLSchema\#string" $>$ aes $</$ Attrib uteValue>

<AttributeDesignator MustBePresent="false"

Category="urn:oasis:names:tc:xacml:3.0:attribute-

category:environment"

AttributeId="http://kth.se/ics/commencryptiontype"

DataType="http://www.w3.org/2001/XMLSchema\#string"/> $<$ /Match $>$

$<$ Match MatchId="urn:oasis:names:tc:xacml:3.0:function:stringequal-ignore-case" $>$ $<$ AttributeValue

DataType="http://www.w3.org/2001/XMLSchema\#string">100</Attrib uteValue>

$<$ AttributeDesignator MustBePresent="false"

Category="urn:oasis:names:tc:xacml:3.0:attribute-category:resource"

AttributeId="http://kth.se/ics/departmentid"

DataType="http://www.w3.org/2001/XMLSchema\#string"/>

$</$ Match $>$

$</$ AllOf $>$

$\langle/$ AnyOf $>$

$</$ Target $>$

$<$ Condition $>$

$<$ Apply FunctionId="urn:oasis:names:tc:xacml:1.0:function:and"> $<$ Apply

FunctionId="urn:oasis:names:tc:xacml:1.0:function:integer-greaterthan-or-equal" >

$$
<\text { Apply }
$$

FunctionId="urn:oasis:names:tc:xacml:1.0:function:string-bag-size"> <AttributeDesignator MustBePresent="false"

Category="urn:oasis:names:tc:xacml:1.0:subject-category:access-

subject" AttributeId="http://kth.se/ics/ipaddress"

DataType="http://www.w3.org/2001/XMLSchema\#string"/> $</$ Apply>

$<$ AttributeValue

DataType="http://www.w3.org/2001/XMLSchema\#integer" $>1</$ Attribu teValue $>$

$<$ Apply>

$<$ Apply FunctionId="urn:oasis:names:tc:xacml:1.0:function:and"> $<$ Apply

FunctionId="urn:oasis:names:tc:xacml:1.0:function:and" $>$ $<$ Apply

FunctionId="urn:oasis:names:tc:xacml:1.0:function:time-greater-thanor-equal"> $<$ Apply

FunctionId="urn:oasis:names:tc:xacml:1.0:function:time-one-andonly">

$$
<\text { AttributeDesignator }
$$

AttributeId="urn:oasis:names:tc:xacml:1.0:environment:current-time" Category="urn:oasis:names:tc:xacml:3.0:attributecategory:environment" 
DataType="http://www.w3.org/2001/XMLSchema\#time"

MustBePresent="true"/>

$</$ Apply>

$<$ AttributeValue

DataType="http://www.w3.org/2001/XMLSchema\#time">08:00:00</A ttributeValue>

$$
\begin{aligned}
& \text { </Apply> } \\
& \text { <Apply }
\end{aligned}
$$

FunctionId="urn:oasis:names:tc:xacml:1.0:function:time-less-than-orequal">

$$
<\text { Apply }
$$

FunctionId="urn:oasis:names:tc:xacml:1.0:function:time-one-andonly">

\section{$<$ AttributeDesignator}

AttributeId="urn:oasis:names:tc:xacml:1.0:environment:current-time" Category="urn:oasis:names:tc:xacml:3.0:attribute-

category:environment"

DataType="http://www.w3.org/2001/XMLSchema\#time"

MustBePresent="true"/>

$$
<\text { Apply> }
$$

$<$ AttributeValue

DataType="http://www.w3.org/2001/XMLSchema\#time" >18:00:00</A ttributeValue>

$$
\begin{aligned}
& \text { </Apply> } \\
& \text { </Apply> } \\
& \text { <Apply }
\end{aligned}
$$

FunctionId="urn:oasis:names:tc:xacml:1.0:function:and"> $<$ Apply

FunctionId="urn:oasis:names:tc:xacml:1.0:function:integer-greaterthan-or-equal">

$$
<\text { Apply }
$$

FunctionId="urn:oasis:names:tc:xacml:1.0:function:integer-one-andonly">

$$
<\text { AttributeDesignator }
$$

Category="urn:oasis:names:tc:xacml:1.0:subject-category:accesssubject" AttributeId="http://kth.se/ics/maxpayload"

DataType="http://www.w3.org/2001/XMLSchema\#integer"

MustBePresent="false"/>

$$
\begin{aligned}
& \text { </Apply> } \\
& \text { <Apply }
\end{aligned}
$$

FunctionId="urn:oasis:names:tc:xacml:1.0:function:integer-one-andonly">

$$
<\text { AttributeDesignator }
$$

Category="urn:oasis:names:tc:xacml:3.0:attribute-category:resource" AttributeId="http://kth.se/ics/payload"

DataType="http://www.w3.org/2001/XMLSchema\#tinteger"

MustBePresent="false"/>

$$
\begin{aligned}
& \text { </Apply> } \\
& \text { </Apply> }
\end{aligned}
$$$$
<\text { Apply }
$$

FunctionId="urn:oasis:names:tc:xacml:1.0:function:integer-greater-

than-or-equal">

$$
<\text { Apply }
$$

FunctionId="urn:oasis:names:tc:xacml:1.0:function:string-bag-size"> <AttributeDesignator MustBePresent="false"

Category="urn:oasis:names:tc:xacml:1.0:subject-category:access-

subject" AttributeId="http://kth.se/ics/clearancecheck"

DataType="http://www.w3.org/2001/XMLSchema\#string"/> $<$ Apply> $<$ AttributeValue

DataType="http://www.w3.org/2001/XMLSchema\#integer">1</Attribu teValue>

$$
</ \text { Apply }>
$$

$</$ Apply>

$</$ Apply $>$

$</$ Apply $>$

$</$ Condition $>$

$</$ Rule $>$

\begin{tabular}{|c|c|c|}
\hline Defined & Attribute & Description \\
\hline Request & $\begin{array}{l}\text { http://kth.se/ics/ } \\
\text { commencryptiontype }\end{array}$ & $\begin{array}{l}\text { Encryption type of the } \\
\text { communication channel }\end{array}$ \\
\hline Request & $\begin{array}{l}\text { http://kth.se/ics/ } \\
\text { departmentid }\end{array}$ & Object's department \\
\hline Policy & $\begin{array}{l}\text { http://kth.se/ics/ } \\
\text { ipaddress }\end{array}$ & $\begin{array}{l}\text { The result of SQL } \\
\text { defined in Appendix C- } \\
1 \text { deciding if the subject } \\
\text { is internally located }\end{array}$ \\
\hline Policy & $\begin{array}{l}\text { http://kth.se/ics/ } \\
\text { maxpayload }\end{array}$ & $\begin{array}{l}\text { The maximum payload } \\
\text { that the robotic arm can } \\
\text { handle. The value is } \\
\text { fetched from the } \\
\text { attribute repository via } \\
\text { PIP with the SQL } \\
\text { defined in Appendix C- } \\
2\end{array}$ \\
\hline Policy & $\begin{array}{l}\text { http://kth.se/ics/ } \\
\text { clearancecheck }\end{array}$ & $\begin{array}{l}\text { The result of SQL } \\
\text { defined in Appendix C- } \\
3 \text { which decides if the } \\
\text { subject's clearance level } \\
\text { is higher than or equal } \\
\text { to the object's } \\
\text { classification level }\end{array}$ \\
\hline Request & http://kth.se/ics/payload & Actual payload \\
\hline Request & $\begin{array}{l}\text { urn:oasis:names:tc:xacml:1.0: } \\
\text { subject:subject-id }\end{array}$ & User ID of the subject \\
\hline Request & $\begin{array}{l}\text { urn:oasis:names:tc:xacml:1.0: } \\
\text { resource:resource-id }\end{array}$ & Asset ID of the object \\
\hline Request & $\begin{array}{l}\text { urn:oasis:names:tc:xacml:1.0: } \\
\text { action:action-id }\end{array}$ & Attempted operation \\
\hline Request & $\begin{array}{l}\text { urn:oasis:names:tc:xacml:1.0: } \\
\text { environment:environment-id }\end{array}$ & $\begin{array}{l}\text { Actual IP address of the } \\
\text { subject }\end{array}$ \\
\hline Request & $\begin{array}{l}\text { urn:oasis:names:tc:xacml:1.0: } \\
\text { environment:current-time }\end{array}$ & $\begin{array}{l}\text { Actual time of the } \\
\text { request }\end{array}$ \\
\hline
\end{tabular}

$<$ Rule RuleId="rule2" Effect="Deny"/>

$</$ Policy $>$

\section{APPENDIX C}

1. SELECT IP_ADDRESS FROM INTERNAL_IP_ADDRESS WHERE IP_ADDRESS = '<http://kth.se/ics/ipaddress >' ;
2. SELECT MAX PAYLOAD FROM INSTRUMENT WHERE ASSET_ID ='<urn:oasis:names:tc:xacml:1.0:resource:resourceid $>$ ' ;

3. SELECT ROLE FROM EMPLOYEE WHERE USER_ID ='<urn:oasis:names:tc:xacml:1.0:subject:subject-id>'AND EXISTS (SELECT * FROM INSTRUMENT WHERE EMPLOYEE.CLEARANCE_LEVEL >= INSTRUMENT.CLASSIFICATION AND ASSET_ID ='<urn:oasis:names:tc:xacml:1.0:resource:resource-id>') ;

\section{APPENDIX D}

\section{REFERENCES}

[1] ICS-CERT, "ICS-CERT Year in Review 2012." 2012.

[2] ICS-CERT, "ICS-CERT Year in Review 2013." 2013.

[3] U.S. Department of Homeland Security, "Common Cybersecurity Vulnerabilities in ICS." May-2011.

[4] ICS-CERT, "ICS-CERT Monitor between September 2014-February 2015.”2015.

[5] GE Measurement \& Control Solutions, "Top 10 Cyber Vulnerabilities for Control Systems.” 2012.

[6] National American Reliability Council, "Top 10 Vulnerabilities of Control Systems and their Mitigations.pdf." Dec-2006.

[7] M. Bishop, Introduction to computer security. Boston: Addison-Wesley, 2005.

[8] L. Janczewski and A. M. Colarik, Eds., Cyber warfare and cyber terrorism. Hershey: Information Science Reference, 2008.

[9] R. S. Sandhu and P. Samarati, “Access control: principle and practice," Commun. Mag. IEEE, vol. 32, no. 9, pp. 40-48, 1994. 
[10] S. Oh and S. Park, "Task-role-based access control model," Inf. Syst., vol. 28, no. 6, pp. 533-562, 2003.

[11] R. Sandhu, "Access control: The neglected frontier," in Information Security and Privacy, 1996, pp. 219-227.

[12] V. C. Hu, D. Ferraiolo, R. Kuhn, A. Schnitzer, K. Sandlin, R. Miller, and K. Scarfone, "Guide to Attribute Based Access Control (ABAC) Definition and Considerations," National Institute of Standards and Technology, NIST SP 800-162, Jan. 2014.

[13] L. Pietre-Cambacedes, M. Tritschler, and G. N. Ericsson, "Cybersecurity Myths on Power Control Systems: 21 Misconceptions and False Beliefs," IEEE Trans. Power Deliv., vol. 26, no. 1, pp. 161-172, Jan. 2011.

[14] A. Valenzano, "Industrial Cybersecurity: Improving Security Through Access Control Policy Models," IEEE Ind. Electron. Mag., vol. 8, no. 2, pp. 6-17, Jun. 2014.

[15] M. Cheminod, L. Durante, L. Seno, and A. Valenzano, "On the description of access control policies in networked industrial systems" in Factory Communication Systems (WFCS), 2014 10th IEEE Workshop on, 2014, pp. 1-10.

[16] K. Stouffer, J. Falco, and K. Scarfone, "NIST, Special Publication 800-82, Guide to Industrial Control Systems (ICS) Security." Jun-2011.

[17] "ISA99, Industrial Automation and Control Systems Security - ISA." [Online]. Available: https://www.isa.org/isa99/. [Accessed: 04-Apr-2015].

[18] M. Onori and J. Barata, "Evolvable Production Systems: new applications in mechatronic equipment", Transactions on Industrial Electronics, IEEE Journal, IES Society, 2010.

[19] WSO2 Identity Server 5.0.0. www.wso2.org: WSO2, 2015.

[20] MySQL Community Server 5.6.24. www.mysql.com: MySQL, 2015.

[21] SoapUI 5.2. www.soapui.com: SmartBear, 2015.

[22] U.S. Department of Homeland Security, "Control Systems Communications Encryption Primer" Dec-2009.

[23] U.S. Department of Energy "Communications Requirements of Smart Grid Technologies" Oct-2010.

\section{Authors' Profiles}

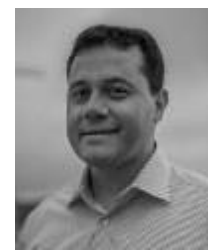

Erkan Yalcinkaya is an independent industrial Ph.D. candidate at Department of Production Engineering, Royal Institute of Technology, Stockholm, Sweden. Erkan is an experienced IT security specialist focused on access control technologies. His main research focus is security aspects of industrial manufacturing systems and production lines.

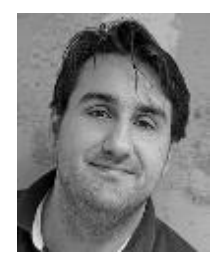

Antonio Maffei received the B.E. and the M.E. degree in industrial engineering from the University of Pisa, Tuscany, Italy. Antonio received a Ph.D. degree in production engineering from $\mathrm{KTH}$, Royal Institute of Technology in Stockholm, Sweden, in 2012.

After a postdoc within the Swedish strategic XPRES initiative, he started a tenure track with an assistant professorship on "Production System focus area business models" at KTH. Dr. Maffei is currently Head of the research group named Technologies for Adaptable Production. His current research interests include business models for advanced automation technology, assembly technology and engineering education. Dr. Maffei is a Research Affiliate of The International Academy for Production Engineering; active in a number of national and international networks.

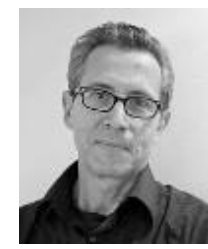

Professor Mauro Onori is the current Head of Department of Production Engineering at the Royal Institute of Technology, School of Industrial Eng. \& Management. Prof. Onori has published over 150 articles, has been European Project Leader since 2006 with participation since 1999, including projects ERace, EUPASS, AssemblyNet, IDEAS, openMOS, etc. Prof. Onori has also been guest lecturer at both École Polytechnique Fédérale de Lausanne and the New University of Lisbon. Onori has also received several awards including the Japan Robot Association Award.

How to cite this paper: Erkan Yalcinkaya, Antonio Maffei, Mauro Onori,"Application of Attribute Based Access Control Model for Industrial Control Systems", International Journal of Computer Network and Information Security(IJCNIS), Vol.9, No.2, pp.12-21, 2017.DOI: 10.5815/ijcnis.2017.02.02 